\title{
TEST PROBLEMS IN RADIATIVE TRANSFER CALCULATIONS
}

\author{
A. I. Shestakov \\ D. S. Kershaw \\ G. B. Zimmerman
}

This paper was prepared for submittal to the Topical Meeting on Advances in Nuclear

Engineering Computation and Radiation Shielding in Santa Fe, New Mexico, April 9-13, 1989.

Also submitted to Nuclear Science and Engineering.

January 12, 1989

This is a preprint of a paper Intended ror publication in a Journal or proceedings. Since changes may be made before publication. this preprint is made arailable with the understanding that It will not be cited or reproduced without the permission of the author.

\section{DISCLAIMER}

This report was prepared as an account of work sponsored by an agency of the United States Government. Neither the United States Government nor any agency thereof, nor any of their employees, makes any warranty, express or implied, or assumes any legal liability or responsibility for the accuracy, completeness, or usefulness of any information, apparatus, product, or process disclosed, or represents that its use would not infringe privately owned rights. Reference herein to any specific commercial product, process, or service by trade name, trademark, manufacturer, or otherwise does not necessarily constitute or imply its endorsement, recommendation, or favoring by the United States Government or any agency thereof. The views and opinions of authors expressed herein do not necessarily state or reflect those of the United States Government or any agency thereof.
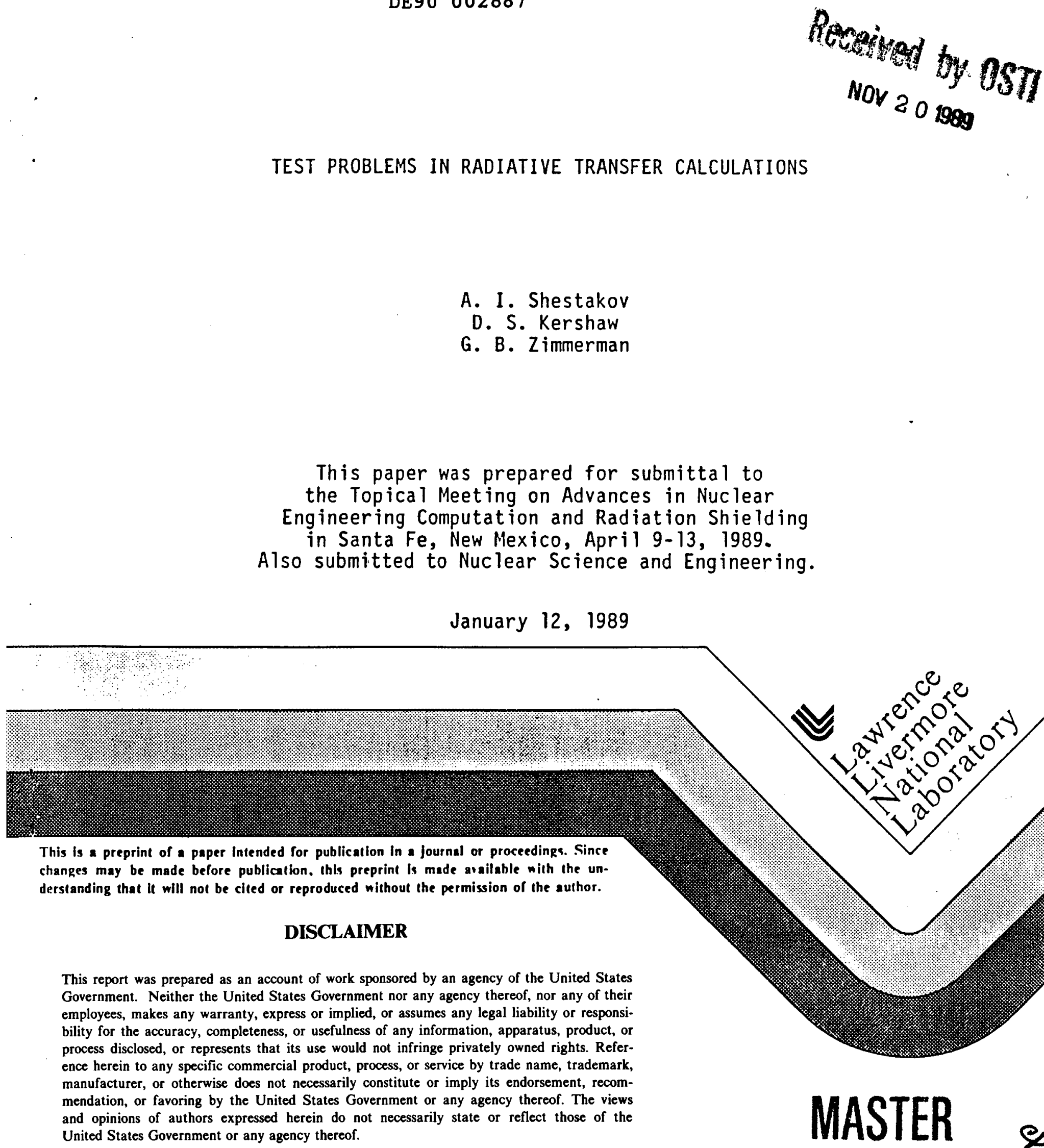


\section{DISCLAIMER}

This report was prepared as an account of work sponsored by an agency of the United States Government. Neither the United States Government nor any agency Thereof, nor any of their employees, makes any warranty, express or implied, or assumes any legal liability or responsibility for the accuracy, completeness, or usefulness of any information, apparatus, product, or process disclosed, or represents that its use would not infringe privately owned rights. Reference herein to any specific commercial product, process, or service by trade name, trademark, manufacturer, or otherwise does not necessarily constitute or imply its endorsement, recommendation, or favoring by the United States Government or any agency thereof. The views and opinions of authors expressed herein do not necessarily state or reflect those of the United States Government or any agency thereof. 


\section{DISCLAIMER}

Portions of this document may be illegible in electronic image products. Images are produced from the best available original document. 
UCRL-99450

\title{
Test Problems in Radiative Transfer Calculations*
}

\author{
A. I. Shestakov, D. S. Kershaw and G. B. Zimmerman \\ Computational Physics Division \\ Lawrence Livermore National Laboratory \\ P.O. Box 5508, Livermore, CA 94550
}

\begin{abstract}
Several test problems are presented for evaluating the radiation diffusion equations. For spatial transport schemes, 1-D problems with known analytic solutions are tested on 2-D domains with non-orthogonal meshes. It is shown that a scheme based on the Finite Element Method is insensitive to grid distortions when the diffusion term is dominant. Other test problems deal with Compton scattering, specifically the 1-D Fokker-Planck equation coupled to an equation describing the change in electron temperature. The test problems model the evolution of a Planckian radiation field as it equilibrates with the electrons. In all cases, the numerical results are compared with the analytic ones.
\end{abstract}

\section{Introduction}

We present a collection of test problems for the equations of radiative transfer in the diffusion approximation ${ }^{1}$. The spectral energy density of the radiation field, $u$, is coupled to the electron temperature, $T_{e}$. The independent variables are the cylindrical coordinates, $R$ and $Z$, the time, $t$, and (for $u$ ) the photon frequency, $v$. The package is incorporated into a code driven by Lagrangian hydrodynamics with distinct ion and electron temperatures. In our applications, the initial $(R, Z)$ grid is usually non-orthogonal, but is specified by logically rectangular $(K, L)$ coordinates. Subsequent Lagrangian motion distorts the mesh. The frequency space is divided into $N_{v}$ groups. We are interested in numerical methods that are stable, conservative, and yield answers independent of mesh distortions or discretizations.

Although the test problems were devised in order to check various radiation transport subroutines, they are applicable to other fields, e.g. solution of parabolic or elliptic equations or the 1-D Fokker-Planck equation. The equations of interest are

$$
\begin{aligned}
\partial_{t} u & =\nabla \cdot D \nabla u+c \sigma_{a}(B-u)+S_{0,}\left(u, T_{e}\right), \\
c_{v} \partial_{t} T_{a} & =-\int d v c \sigma_{a}(B-u)-\int d v S_{\sigma_{s}}\left(u, T_{e}\right) .
\end{aligned}
$$

Equations (1) and (2) respectively represent the Lagrangian time derivative of the radiation and electron energies; $c_{v}$ is the electron specific heat. The first term on the rhs of Eq.(1) describes the spatial transport of $u$; the transport diffusion coefficient $D$ varies both spatially and with frequency through its dependence on the absorption, $\sigma_{a}$, and scattering, $\sigma_{s}$, coefficients. Equation (1) is an approximation to the radiation transfer equation and is valid whenever the radiation field is nearly isotropic ${ }^{1.2}$. However, suitably modified, the diffusion equations give acceptable results in other problems. One such change alters $D$ by introducing a dependence on $\nabla u$ to limit the speed of propagation of $u$ to that of light (i.e. a flux-limiter).

* Work performed under the auspices of the United States Department of Energy by the Lawrence Livermore National Laboratory under contracl number W-7405-ENG-48. 
The dependence on matter properties is displayed by the remaining terms of Eq.(1). Radiation emission, given by the Planck function, $B_{v}\left(T_{e}\right)$, and absorption are represented by the second term in Eq.(1). The last term describes the energy exchange due to Compton scattering and usually is modeled by a diffusion equation in frequency space, the Compton Fokker-Planck approximation. Equation (2) exhibits energy conservation. Energy losses of the radiation field are electron energy sources.

Problems to evaluate diffusion schemes are presented in Secs. 2, 3 and 4. Section 2 considers linear diffusion ( $D=$ const) while Sec. 3 describes a flux-limited problem. Section 4 introduces strong-matter coupling; the large $\sigma_{a}$ value reduces to a non-linear diffusion equation for $T_{e}$, the thermal wave. Section 5 is restricted to Compton scattering. First we present a numerical method that solves the coupled system of equations for $u$ and $T_{e .}$. Subsequently (Secs. 5.1 and 5.2), the scheme is applied to problems with known analytic solutions. The problems model the evolution of an initially Planckian field surrounded by electrons at a disparate temperature.

\section{Linear Diffusion}

In the first collection of problems, we evaluate schemes for modeling both linear and flux-limited diffusion. Setting $\sigma_{a}$ and $S=0$, and $N_{\mathrm{v}}=1$ gives a single parabolic equation for $u$. Kershaw proposed a finite difference method (FD) based on zone centered quantities ${ }^{3}$. Such zonal schemes are now commonly used on Lagrangian meshes; they compute the intuitively reasonable, average energy of the material in a zone. However, we find that finite element methods (FE) are considerably more accurate and less sensitive to grid distortions.

The application of FE to Eq.(1) is described in reference 4. Examples and comparisons appear in references 4 and 5. Here we synopsize and extend those results. Briefly, FE is implemented by bisecting each Lagrangian quadrilateral into triangles and representing $\boldsymbol{u}$ in terms of piecewise-linear basis functions. The numerical solution is specified by its values on the grid points, i.e. a nodal representation. The methods are tested by solving diffusion equations on a 2-D domain with initial and boundary conditions that dictate a 1-D solution. The numerical grid (which is fixed in all our test problems) is deliberately skewed. Thus, deviations in the numerical solution from the expected symmetry, are due strictly to the numerical method and thereby gauge its accuracy.

Comparisons are made on two different meshes. The first, "random" mesh, Fig.1, models the Lagrangian hydrodynamic distortions that may arise in a simulation. The FORTRAN program generating the mesh is available from the authors and is listed in Appendix A. On such meshes, FD is expected to show grid effects since the method's accuracy depends on a smooth mapping between the physical $R, Z$ and the logical $K, L$ variables. To compare FD and FE on grids with a smooth $R, Z$ to $K, L$ Jacobian, a square domain is discretized by an analytic function which constructs the mesh ${ }^{5}$, Fig.2,

$$
R=s+\alpha \sin (\pi s) \cos (\pi t) \text {, and } Z=t-\alpha \sin (\pi t) \cos (\pi s) \text {. }
$$

If $\alpha \leq 1 / \pi$, then for $0 \leq s, t \leq 1$, Eq.(3) implies $0 \leq R, Z \leq 1$. Given two integers $K M A X$ and $L M A X$, the mesh is generated by the arrays, $\left[s_{L}\right]_{L=1}^{L M A X}$ and $\left.\left[t_{X}\right]\right]_{X=1}^{K M A X}$ where $s_{L}=(L-1) /(L M A X-1)$ and $t_{K}=(K-1) /(K M A X-1)$. For the test problems, the random and "smooth" meshes are translated by one in the $R$ direction. We add an extra row of zones above and below both grids in order to impose the boundary conditions.

The grids are used to solve the energy conduction equation,

$$
\partial_{1} u=(1 / R) \partial_{R}\left(R D \partial_{R} u\right)
$$

on the domain: $R_{0} \leq R \leq R_{1}$ and $0 \leq Z \leq 1 *$. The diffusion coefficient is a function of the mean free path, $l$,

$$
D=c l / 3 \text {. }
$$

In this section, we fix $l=10^{-2}$. As initial condition, we set $u=1.372 \cdot 10^{-26}$. A Milne or mixed boundary condition,

\footnotetext{
- We purposely consider diffusion in $R$ instead of $Z$ in order not to give FE an unfair advantage. When $R$ is the independent variable, the steady-state solution depends on $\ln (R)$. Otherwise, the steady-state solution would be a linear function of $Z$ and since we use piecewise-linear elements, FE is guaranteed to get the exact solution.
} 


$$
u+(2 / 3) \partial_{R} u=0,
$$

is imposed along $R_{1}$. Symmetry conditions hold along the $Z$ boundaries. At time $t=0$ and thereafter we fix $u=u_{0}=7.042 \cdot 10^{-13}$ along $R=R_{0}$. The boundary conditions imply that the equilibrium solution is:

$$
u_{2}=u_{0} \frac{1+\left(3 R_{1} / 2 l\right) \ln \left(R_{1} / R\right)}{1+\left(3 R_{1} / 2 l\right) \ln \left(R_{1} / R_{0}\right)} \text {. }
$$

The outgoing energy flux along $R=R_{1}$ is $-D \nabla u=(c / / 3) \partial_{R} u_{\varepsilon}$.

Any variation in the numerical solutions with $Z$ is due to discretization errors. We examine these by plotting the equilibrium energies along the outgoing boundary. The zonal and nodal nature of the two schemes implies that the boundary conditions are applied at different values of $R_{0} ;$ FE uses $R_{0}=1$, while FD uses $R_{0}=0.9845$. The numerical results are in terms of the energy per gram and, hence, should be multiplied by the density $\left(=10^{2}\right)$. Furthermore, the results give the average energy in the last zone, or equivalently at $R=\tilde{R}_{1}=2.01567$. Using Eq.(7), the exact values are $1.0862 \cdot 10^{-14}$ (for FE) and $1.0692 \cdot 10^{-14}$ (for FD). Figure 3 plots the energies as a function of $Z$. Note the greater variation in the FD results. We quantify the errors by computing the difference of the extrema of the numerical results along the last row divided by the exact analytic value,

$$
e_{u} \equiv\left(u_{\max }-u_{\min }\right) / u_{e} \text {. }
$$

Let $F D$ and $F E$ resp. denote the results of the corresponding scheme, and $r$ and $s$ resp. denote the results on the random or smooth meshes. We find

$$
e_{u}(F D, r)=0.127, \quad e_{u}(F E, r)=1.166 \cdot 10^{-3}, \quad e_{u}(F D, s)=0.0451, \quad e_{u}(F E, s)=7.089 \cdot 10^{-4} .
$$

The contrast is even more dramatic if we examine the outgoing equilibrium fluxes. Using Eq.(7), the exact values are $4.8696 \cdot 10^{-13}$ (for FE) and $4.7650 \cdot 10^{-13}$ (for FD). Figure 4 displays the fluxes for both schemes as a function of $Z$. Using Eq.(8), we define $e_{f}$ as the error in the fluxes, then

$$
e_{\lambda}(F D, r)=1.092, \quad e_{f}(F E, r)=2.26 \cdot 10^{-3}, \quad e_{\mathcal{N}}(F D, s)=0.110, e_{f}(F E, s)=1.44 \cdot 10^{-3} .
$$

The results in Eqs. (9) and (10) clearly display the superiority of FE.

\section{Flux-Limited Diffusion}

The diffusion equation is characterized by an infinite speed of propagation. The characteristic diffusion equilibration time, $t_{D}$, may be estimated by dimensional analysis of Eq.(4)

$$
u / t_{D}=D u / d^{2} \text { hence, } t_{D}=d^{2} / D \text {, }
$$

where $d(=1)$ is the characteristic gradient length of the problem. Define the light transit time $t_{c} \equiv d / c$. In this section, we consider $l=10^{2}$. If $D$ is defined by Eq.(5), then $t_{c}>t_{D}$, since $l>3 d$; which has the unphysical implication that the problem relaxes to equilibrium faster than the time for light to travel across the domain. In such cases, characterized by large $l$ values, $D$ is modified by introducing a flux-limiter to limit the speed of signal propagation. Entire papers have been written on the proper choice for a flux-limiter ${ }^{6}$. Here we use the most elementary form, viz.

$$
D=c / \max (3 / l,|\nabla u| / u),
$$

and implement it explicitly.

The initial and boundary conditions for the problem are the same as in the previous section. The only exception is that for FD, $R$ ranges between 1 and 2 which implies that $u$ is fixed along $R_{0}=1.0157$ and Eq.(6) is imposed at $R_{1}=2$. This implies that the equilibrium solutions for both schemes are also given by Eq.(7) and are independent of the flux-limiter since $\max (|\nabla u| / u)<3 / l$.

The efficacy of the flux limiter and the scheme's mesh dependence is demonstrated in Table 1. where we give values for the extrema of $u$ along $R_{1}$ at a time approximately corresponding to $3 / 4$ of the light transit time. The results underscore the shortcomings of diffusion theory as the source should not yet be felt at the outgoing boundary. Nevertheless, the larger variations of FD further evidence FE's superiority for diffusion problems. It is noteworthy that FE gives similar answers on both meshes (cf. rows 4 and 5 of Table 1). FD, on the other 
hand, shows tremendous differences (cf. rows 2 and 3). The flux-limiter, which at this time is dominant, is strongly influenced by the mesh. This is evident in Fig.5 where the contours of $u$ of FD are displayed and contrasted by the corresponding FE contours of Fig. 6 .

The equilibrium results are given in Table 2 where we display the extrema of the average energy in the outgoing zones and the outgoing fluxes. As before, the boundary conditions are imposed in slightly different places for the two schemes which implies that the exact values also differ. As for the transients displayed in Table 1, FE's results are considerably less sensitive to the mesh. Furthermore, FD is even unable to get the correct energy value at $R_{1}$ on the random mesh; its $u_{\max }$ and $u_{\text {min }}$ values are $4 \%$ low.

The results of Tables 1 and 2 and Figs. 5 and 6 reaffirm the greater accuracy of FE for transport dominated problems.

\section{Thermal wave}

In many laser fusion applications, $u$ is strongly coupled to $T_{e}$. To study strong matter coupling set $S=0, \sigma_{a}=1 / l, D=c l / 3$, and $N_{v}=1$ in Eqs.(1) and (2). The Planck function is given by

$$
B_{v}\left(T_{e}\right)=a^{\prime} v^{3} /\left(e^{x}-1\right), x \equiv v / T_{e},
$$

where $a^{\prime}$ is a dimensional constant. For constant $l$, the $v$ dependence is eliminated by integrating Eq.(1) over all frequencies. Redefine $u$ as the total radiation energy density, i.e. $\int d v u \rightarrow u$. Using

$$
\int_{0}^{-} d v B_{v}\left(T_{e}\right)=a^{\prime}\left(\pi^{4} / 15\right) T_{e}^{4} \equiv a T_{e}^{4} .
$$

we obtain two coupled equations for $u$ and $T_{a}$. The electron energy equation may be solved for $u$ and the result substituted into the modified radiation equation to get a single (albeit complicated) equation for $T_{c}$. Let $t_{e}$ define a characteristic time for $T_{e}$ to diffuse one unit of length ( $t_{c}$ is quantified below). By making judicious choices for $l, c_{v}$, and the initial value of $T_{e}$, viz.

$$
l<c t_{e}, a^{\prime} T_{e}^{3}<c_{v} \text {, and }\left(H c t_{e}\right) c_{v}<a T_{e}^{3} \text {. }
$$

the resulting $T_{\text {a }}$ equation is of the form,

$$
c_{v} \partial_{t} T_{a}=\nabla \cdot D \nabla\left(a T_{a}^{4}\right)
$$

The first inequality in Eq.(13) implies strong matter coupling, i.e. the diffusion coefficient is dominated by the absortion and emission terms. The second inequality is satisfied whenever the matter energy, $c_{v} T_{a}^{4}$, dominates the radiation energy. The last inequality further stresses that $l \ll 1$. All these approximations are realistic in our applications, characterized by short $l$ 's and $u \approx B_{\mathrm{v}}\left(T_{e}\right)$.

One dimensional versions of Eq.(14) are discussed in reference 7. If $c_{\mathrm{v}}$ and $D$ are constant, self-similar analytic solutions are known for certain geometries and boundary conditions. Specifically, we consider the solution of Eq.(14) in slab symmetry for an instantaneous plane source. The result is a monotonically decreasing function, symmetric about $Z=0$ which drops sharply to zero at the wave front. $Z_{f}$. The speed of $Z_{f}$ decays rapidly with time.

Numerically, we proceed as in reference 4; two coupled equations are solved for both $u$ and $T_{a}$. The choice of $l$ dictates that although $u$ is transported, $T$, evolves according to the thermal wave equation. The equations are solved by the "partial temperature scheme" ${ }^{4}$. As in the previous sections, the equations are solved in a 2-Dimensional domain on both the random and smooth meshes. The boundary and initial conditions dictate a 1-Dimensional result; deviations from symmetry are mesh errors. The problem has several features which require an accurate solution. For example, energy conservation and a positive solution are imperative as the speed of the wave front is proportional to a power of the total energy in the problem and the solution, $T_{a}$. is meaningful only if positive.

The inequalities of Eq.(13) are satisfied by choosing $l=0.001$ and $c_{v}=50$. In our units $c=300$ and the radiation constant $a=0.0137$. Since $D=c / / 3$, the numerical problem models the 1-Dimensional equation,

$$
\partial_{\mathrm{t}} T=\alpha \partial_{\mathrm{Z}}\left(T_{\mathrm{a}}^{3} \partial_{\mathrm{Z}} T\right), \quad \alpha=4 a c / 3 c_{\mathrm{v}}=1.096 \cdot 10^{-4} .
$$

The numerical domain consists of two random or two smooth grids, each of unit length, $2 / 32 \leq Z \leq 1+2 / 32$ 
and $1+3 / 32 \leq Z \leq 2+3 / 32$. The ignorable coordinate, $R$, varies from 1 to 2 . The $0 \leq Z \leq 1 / 32$ domain is discretized uniformly in $R$. Grid lines are drawn to connect the remaining strips. This causes large shear of the grid lines in the two regions: $1 / 32 \leq Z \leq 2 / 32$ and $1+2 / 32 \leq Z \leq 1+3 / 32$. The two straight grid lines (at $Z=$ 1.0625 and 1.09375 ) allow comparison with the analytical solution. Along $Z=2.09375$ we impose the analogue of Eq.(6). However, this has no effect on the solution and any homogeneous condition will work. The numerical solution loses meaning when the wave reaches $Z_{\max }$. The uniform zoning for $Z \leq 1 / 32$ allows the initialization of $u$ and $T$ as numerical nodal or zonal $\delta$-functions with a density independent of $R$. For FD, the average value of $T_{e}=0.5$ in $0 \leq Z \leq 1 / 32$, while for FE, $T_{c}=1$ at $Z=0$. We also set $u=a T_{e}^{4}$ in order to be in thermal equilibrium.

For this problem the two diffusion schemes (FD and FE) performed similarly. Figure 7 displays the $T$. profiles on the random mesh for FE at time $t=6.8146 \cdot 10^{8}$. The three curves plot $T$, along three different grid lines, along the top $(R=2)$, the bottom $(R=1)$, and "middle." The "middle" grid line corresponds to the logical index $K=16$ and in this problem $1 \leq K \leq 33$ and $1 \leq L \leq 68$. The $K=16$ line is a collection of short straight segments which wander in $R$ as the line extends in $Z$. The overlay of the lines shows that the numerical solution is nearly 1-Dimensional. As the problem is self-similar, the profiles of Fig.7 are representative of all time. With our choice of parameters the front position and temperature vary as

$$
Z_{f}=0.018790 t^{1 / 5} \text { and } T=0.98869 t^{-1 / 5}\left[1-\left(Z / Z_{f}\right)^{2}\right]^{1 / 3} \text {. }
$$

Equation (16) implies that for the time of Fig.7, $Z_{\mathcal{G}}=1.09803$. This is within one grid width of the numerical front position displayed.

The characteristic time, $t_{\text {, }}$, of Eq.(13) may be estimated from a dimensional analysis of Eq.(15). Proceeding as in Eq.(11), $t_{e} \approx 9124 /\left\{T_{e}\right\}^{3}$, where $\left[T_{e}\right\}$ is an average temperature, $\left\{T_{e}\right\}<1$ since that is its maximum at $t=0$. As time evolves, $\left\{T_{a}\right\}$ decays; indeed, setting $Z_{f}=1$ in Eq.(16) defines $t_{e} \approx 4.3 \cdot 10^{8}$.

Deviation from the expected symmetry is observed by plotting $T_{e}$ as a function of $R$ for fixed $Z$ at $t \approx 6.8 \cdot 10^{8}$ and $t=2 \cdot 10^{9}$. The times correspond to $Z_{f} \approx 1.098$ and 1.3619 respectively. Thus, we examine $T_{\text {a }}$ just after the front has passed (when we expect the greatest asymmetrical noise) and after $Z_{f}$ is "far away" (when the noise should have dissipated). For FD, we plot the average value for $1.0625 \leq Z \leq 1.09375$, or equivalently at $Z=1.078125$. For $F E$, we plot at $Z=1.09375$. Figure 8 displays $T$, vs. $R$ for $F D$ and $F E$ for both meshes at the early time. Figure 9 has the same plots at the later time. The plots are not at the same time which accounts for the difference in the values. The analytic values are given in Table 3. The larger noise for $\mathrm{FE}$ in Fig. 8 is due to the closer proximity of the points to $Z_{f}$ (FE results at $Z=1.0625$ are smoother). By the later time, the FE results are nearly uniform in $R$, cf. Fig.9. The same behavior is found when comparing the mean values of Figs. 8 and 9 to the analytic values of Table 3.

\section{Compton Scattering}

To evaluate numerical algorithms of this subject we set $D$ and $\sigma_{a}=0$ in Eqs.(1) and (2). The resulting first equation describes the spectral change of $u$ due to its interaction with a scattering medium. The second (electron) equation asserts energy conservation. In the diffusion approximation, the most exact treatment of scattering is given by the Boltzman equation. In this approximation the scattering operator, $S$, is a complicated integral over all frequencies of both $u$ and $T_{e}$. The difficulty associated with solving integral equations has led to further approximation. In particular, by making expansions valid for small $v$ and $T$, Kompaneets replaced the Boltzman integral by a diffusion equation in frequency space ${ }^{8}$. The resulting Compton Fokker-Planck operator shares two important features with Boltzman; it conserves the number of photons and has the same steady-state. The operator derived by Kompaneets has a diffusion coefficient proportional to $v^{4}$. Subsequent authors noticed the rate of energy change of Fokker-Planck differed with Boltzman. This led to modifications of the diffusion coefficient (see reference 9 for citations). The modifications are contained in a nondimensional function $\alpha$ of order 1 which depends on $v$ and $T_{e}$.

Before discussing the test problems, we present the relevant equations and the numerical method which solves them. The implicit time-advancement scheme is due to the third author (G.B.Z.). It is convenient to work in non-dimensional units. Conversion to CGS units is given in reference 9, App. A. We define $\gamma$ and $\tau$ to be the non-dimensional photon frequency and electron temperature, $\gamma \equiv h v / m_{e} c^{2}$ and $\tau \equiv k T / m_{e} c^{2}$. The time variable is normalized by multiplication by $c N_{c} \sigma_{T}$ (the speed of light $\times$ the electron number density $\times$ the Thomson $x$-section). The dependent variables are the photon distribution function, $n$ (proportional to $\mu / v^{3}$ ) and 
$\tau$. The relevant equations are

$$
\begin{aligned}
\gamma^{2} \partial_{r} n & =\partial_{\gamma}\left\{\alpha(\gamma, \tau) \gamma^{4}\left[\tau \partial_{\gamma} n+n(1+n)\right]\right\}, \\
c_{v} \partial_{\imath} \tau & =\int d \gamma\left\{\alpha(\gamma, \tau) \gamma^{4}\left[\tau \partial_{\gamma} n+n(1+n)\right]\right\} .
\end{aligned}
$$

The number of photons and the energy density of the radiation field are respectively defined as

$$
N \equiv \int d \gamma \gamma^{2} n \text { and } E_{r} \equiv \int d \gamma \gamma^{3} n \text {. }
$$

Hence, integrating Eq.(17) over $\gamma$ restates conservation of photon number. Equation (18) is obtained by first multiplying Eq.(17) by $\gamma$ and then integrating by parts. It implies energy conservation; since $c_{\mathrm{v}} \tau$ is identified as the electron energy. Equations (17) and (18) include the effects of stimulated scattering. These may be excluded by replacing the $(1+n)$ term by 1 .

As discussed above, the Fokker-Planck operator has the same equilibrium solution as Boltzman. If stimulated scattering is included, the result is a Bose-Einstein distribution,

$$
n_{B}=1 /\left(C e^{r t}-1\right), C=\text { const } \geq 1 \text {. }
$$

The special case, $C=1$, is a Planck distribution, cf. Eq.(12) and the definition of $E_{r}$ in Eq.(19). If stimulated scattering is excluded, the steady-state is a Wien distribution,

$$
n_{W}=C e^{-r \tau}, C=\text { const. }
$$

Numerical solutions of Eqs.(17) and (18) begin with a discussion of the discretization of the rhs of Eq.(17). We modify the "quasi-integral" method of reference 10 . Using the identity $d e^{\gamma \tau}=(1 / \tau) e^{\gamma \tau} d \gamma$, the square-bracketed term of Eqs.(17) and (18) is rewritten as a single derivative,

$$
\tau \partial_{\gamma} n+n(1+s n)=(1+s n)^{2} \frac{\partial}{\partial e^{\gamma \tau}}\left[\frac{n e^{\gamma \tau}}{1+s n}\right] \equiv F,
$$

where $s$ is a convenient multiplier (= 0 or 1 ) that allows exclusion of stimulated scattering. In this way, if $F=0$, which is true for an equilibrium solution, the term in large parentheses is constant. Hence, if $s=0$, $n=n_{W}$ and if $s=1, n=n_{B}$. The range of frequencies is discretized into $N_{v}$ groups. Let $\gamma_{j}$ and $\Delta_{j}$, $j=1, \cdots, N_{\mathrm{v}}$ respectively denote the group midpoints and group widths. If the subscript denotes the group index, Eq.(17) is discretized as

$$
\begin{gathered}
\gamma_{j}^{2} \Delta_{j}\left(n_{j}-n_{j}^{o}\right)=G_{j+1 / 2}-G_{j-1 / 2}, j=1, \cdots, N_{v}, \\
G_{1 / 2} \equiv 0, G_{N_{v}+1 / 2} \equiv 0, G_{j+1 / 2} \equiv \Delta l \alpha_{j+1 / 2} \gamma_{j+1 / 2}^{4} F_{j+1 / 2}, j=1, \cdots, N_{v}-1,
\end{gathered}
$$

and for $j=1, \cdots, N_{v}-1, \gamma_{j+1 / 2}^{2}$ are the group edges, e.g. $\gamma_{j+1 / 2}^{2} \equiv \gamma_{j} \gamma_{j+1}$. The superscript "o"of Eq.(23) denotes the old time level and $F_{j+1 / 2}$ is a discretization of $F$ in Eq.(22), viz.

$$
\begin{gathered}
F_{j+1 / 2}=\frac{(1+s n)_{j+1 / 2}^{2}}{e^{\gamma_{j+1} / \tau}-e^{\gamma_{j} / \tau}}\left[\frac{n_{j+1} e^{\gamma_{j+1} / \tau}}{(1+s n)_{j+1}}-\frac{n_{j} e^{\gamma_{j} / \tau}}{(1+s n)_{j}}\right]=\frac{1+s n_{j}^{i}}{1-e_{j+1 / 2}} n_{j+1}-\frac{\left(1+s n_{j+1}^{i}\right) e_{j+1 / 2}}{1-e_{j+1 / 2}} n_{j}, \\
e_{j+1 / 2} \equiv \exp \left[-\left(\gamma_{j+1}-\gamma_{j}\right) / \tau\right]
\end{gathered}
$$

The second expression is preferred as it avoids positive exponentials. The " $i$ " superscript is an iteration index, and denotes the best guess for $n_{j}$. For $s=1$, one may iterate for $n_{j}$; at the first pass, or if no iteration is desired, $n_{j}^{i}=n^{0}$. In the second expression for $F_{j+1 / 2}$, we used the interpolation formula, $(1+s n)_{j+1 / 2}^{2}=(1+s n)_{j}(1+s n)_{j+1}$. It is trivial to show that Equation (23) conserves photons. The discrete analogue of the change in photon number is

$$
\Delta N=\sum_{j=1}^{N_{v}} \Delta_{j} \gamma_{j}^{2}\left(n_{j}-n_{j}^{o}\right)=0,
$$

since by construction the rhs is a telescoping sum. Furthermore, the discretization preserves the equilibrium. The rhs of Eq.(23) vanishes if each of the $F_{j+1 / 2}$ vanish. Depending on $s, F_{j+1 / 2}=0$ whenever $n_{j}$ is a discretization of either Eq.(20) or (21). 
The photon fluxes in Eq.(23) may be expressed as a difference of the densities,

$$
\begin{gathered}
G_{j+1 / 2}=a_{j n_{j+1}-b_{j+1} n_{j},} \\
a_{j}=\frac{\Delta t \alpha_{j+1 / 2} \gamma_{j+1 / 2}^{4}\left(1+s n_{j}^{j}\right)}{1-e_{j+1 / 2}}, \quad b_{j+1}=\frac{\Delta t \alpha_{j+1 / 2} \gamma_{j+1 / 2}^{4}\left(1+s n_{j+1}^{i}\right) e_{j+1 / 2}}{1-e_{j+1 / 2}}, j=1, \cdots, N_{v}-1 .
\end{gathered}
$$

Hence, Eq.(23) is a linear tridiagonal system of order $N_{v}$ of the form

$$
\begin{gathered}
\mathrm{A}(\tau) \times(\tau)=y, \text { or } \\
-b_{j} n_{j-1}+\left(\gamma_{j}^{2} \Delta_{j}+a_{j-1}+b_{j+1}\right) n_{j}-a_{j} n_{j+1}=\gamma_{j}^{2} \Delta_{j} n_{j}^{o}, j=1, \cdots, N_{v}, \\
\text { with } a_{0}=b_{1}=a_{N_{v}}=b_{N_{v}+1}=0 .
\end{gathered}
$$

Equation (24a) explicitly states that the matrix $A$ and the unknown vector $x$ depend on the new electron temperature, $\tau$. The dependence is through the coefficients $a_{j}$ and $b_{j}$. As discussed in reference 10 , the nonnegativity of the terms in Eq.(24b) implies that the system is diagonally dominant, hence its solution is nonnegative.

The equilibrium solution strongly depends on $\tau$, see Eqs.(20) and (21), which implies the same for the transients and so it behooves to have the time advancement implicit in $\tau$. To this end, $\tau$ is expressed as

$$
\tau=\tau^{o}+\Delta \tau \text {. }
$$

The matrix and the new distribution are linearized with respect to $\tau$.

$$
\begin{gathered}
\mathbf{A}(\tau)=\mathbf{A}\left(\tau^{0}\right)+\mathbf{A}^{\prime} \Delta \tau, \quad \mathbf{A}^{\prime}=d \mathbf{A} /\left.d \tau\right|_{\tau-\tau^{\circ}} \\
\text { and } \mathbf{x}(\tau)=\mathbf{x}\left(\tau^{o}\right)+\mathbf{x}^{\prime} \Delta \tau .
\end{gathered}
$$

Substituting into Eq.(24a), collecting like quantities, and dropping the $O\left(\Delta \tau^{2}\right)$ term gives two linear systems for the unknowns $x\left(\tau^{\circ}\right)$ and $x^{\prime}$,

$$
\begin{gathered}
A\left(\tau^{\circ}\right) \mathbf{x}\left(\tau^{\circ}\right)=y \quad \text { and } \\
A\left(\tau^{\circ}\right) x^{\prime}=-A^{\prime} \mathbf{x}\left(\tau^{\circ}\right) .
\end{gathered}
$$

The two systems are solved in succession. Only $\mathbf{A}\left(\tau^{\circ}\right)$ needs to be inverted, hence only one LU decomposition is needed.

Equation (26b) shows that $x(\tau)$ is a function of $\Delta \tau$. The temperature change is obtained from energy conservation. Let $n_{j}\left(\tau^{o}\right)$ and $n_{j}^{\prime}$ denote the elements of $\mathbf{x}(\tau)$ and $\mathbf{x}^{\prime}$ resp. Then,

$$
\Delta E_{r}=\sum_{j=1}^{N_{v}} \Delta_{j} \gamma_{j}^{3}\left(n_{j}\left(\tau^{o}\right)+n_{j}^{\prime} \Delta \tau-n_{j}^{o}\right)
$$

represents the energy change in the radiation field. The negative of this is the electron energy change and gives the $\Delta \tau$ equation, i.e. $\Delta E_{e}=c_{v} \Delta \tau=-\Delta E_{r}$. Hence,

$$
\left.\Delta \tau=-\left[\sum \Delta_{j} \gamma_{j}^{3}\left(n_{j}\left(\tau^{\circ}\right)-n_{j}^{o}\right)\right] /\left[c_{\mathrm{v}}+\sum \Delta_{j} \gamma_{j}^{3} n_{j}^{\prime}\right)\right] .
$$

By construction, energy is conserved. The temperature is given by Eq.(25) and the new radiation field by Eq.(26b).

Although the above scheme performed well on the test problems, it has one unfortunate feature. The new density $\mathbf{x}(\tau)$ is not necessarily positive. Equation $(26 \mathrm{~b})$ states that $\mathbf{x}(\tau)$ is a sum of two vectors. The first, $\mathbf{x}\left(\tau^{\circ}\right)$, is positive since it solves Eq.(27a) and that rhs is positive. However, the $j^{\text {th }}$ term on the rhs of Eq.(27b) is analogous to a divided difference approximation of $\Delta \Delta_{j} \partial_{\gamma}\left(\alpha \gamma^{4} \partial_{\gamma} n\right)$. The latter term is of indeterminate sign. Hence the sign of the components of $x^{\prime}$ is also indeterminate and for large $\Delta \tau$, Eq.(26b) implies that some of the $x_{j}(\tau)$ may be negative. However (ignoring round-off), $N$ is conserved. It is straightforward to show that the sum $\Sigma\left(A^{\prime} \mathbf{x}\left(\tau^{\circ}\right)\right)_{j}=0$. Thus, since $x^{\prime}$ solves Eq.(27b), and since $A$ is conservative, the "total number" of photons in $\mathbf{x}^{\prime}=0$. 


\subsection{Wien Equilibria}

In this and the following section we model the time evolution to steady state of a photon gas interacting with Maxwellian electrons. We assume that the electrons obey an ideal gas law, i.e. $c_{\mathrm{v}}=$ const. Initially, the radiation field has a Planckian distribution with a temperature $\theta_{0}$ and the electrons are at temperature $\tau_{0}$. Equation (20) implies that

$$
n(t=0)=\left(e^{\gamma / \theta_{0}}-1\right)^{-1}
$$

We examine the equilibrium solution and the rate of energy change. Both up and down scatter is considered. For the former, $\tau_{0}>\theta_{0}$; the photons gain energy from the electrons. In down scatter, $\tau_{0}<\theta_{0}$; the photons lose energy.

In this section stimulated scattering is ignored. The final distribution is given by Eq.(21) which is characterized by two constants, $C$ and $\tau$. For the analysis we need the following:

$$
\int_{0}^{\infty} d \gamma \gamma^{m} e^{-\gamma / \theta}=m ! \theta^{m+1} \text { and } \int_{0}^{\infty} d \gamma \gamma^{m} /\left(e^{\gamma / \theta}-1\right)=m ! \zeta(m+1) \theta^{m+1},
$$

where $\zeta$ is the Riemann zeta function "1. Recall the definitions of $N$ and $E_{r}$ in Eq.(19). Equations (21) and (29) imply that photon number conservation may be expressed as

$$
2 ! \zeta(3) \theta_{0}^{3}=2 ! C \tau^{3} \text {. }
$$

Equations (28), (29) and energy conservation imply

$$
c_{v} \tau_{0}+3 ! \zeta(4) \theta_{0}^{4}=c_{v} \tau+3 ! C \tau^{4} .
$$

Given $c_{v}$, the equations are easily solved,

$$
C=\zeta(3)\left(\theta_{d} \tau\right)^{3}, \quad \tau=\left[c_{v} \tau_{0}+6 \zeta(4) \theta_{0}^{4}\right] /\left[c_{v}+6 \zeta(3) \theta_{0}^{3}\right] .
$$

At the end of the run, our program computes a "radiation temperature", $\theta$, assuming that the photon distribution is Planckian. However, in this section, $n_{W}$ is the final photon distribution. Equations (21) and (29) imply that $\theta$ and $C$ are related, since we may equate the energies,

$$
3 ! C \tau^{4}=3 ! \zeta(4) \theta^{4} \quad \text { or } \quad \theta=[C / \zeta(4)]^{1 / 4} \tau .
$$

Thus, by comparing the numerical $\theta$ with Eq.(33) we may check that $N$ is conserved. Another check compares the maximum of the energy density with the numerical value

$$
\left(\gamma^{3} n_{W}\right)_{\max }=C(3 \tau / e)^{3} \text { at } \gamma=\gamma_{W, \max }=3 \tau .
$$

When stimulated scattering is neglected, the rate of energy change

$$
\frac{d E_{r}}{d t}=\int_{0}^{\infty} d \gamma \gamma^{3} n(\gamma, t) R(\gamma, \tau)
$$

where $R$ is a certain integral of the Compton scattering kernel, see references 12 and 9 . The results of reference 12 show that $R(\tau<1)=-\gamma \alpha_{0}(\gamma)+\tau \alpha_{1}(\gamma)$ where $\alpha_{0}$ and $\alpha_{1}$ are known analytic functions of $\gamma$. Substituting the power series expansions for the $\alpha_{i}$ (valid for small $\gamma$ ) and the distribution given in Eq.(28) into Eq.(35), gives an expression which may be integrated term by term.

$$
\frac{1}{4 E_{r}} \frac{d E_{r}}{d t}=\left[\tau-\frac{\zeta(5)}{\zeta(4)} \theta_{0}\right]-\theta_{0}\left[\frac{47 \zeta(5)}{2 \zeta(4)} \tau-\frac{21 \zeta(6)}{\zeta(4)} \theta_{0}\right]+\frac{\theta_{0}^{2}}{2}\left[\frac{567 \zeta(6)}{\zeta(4)} \tau-\frac{441 \zeta(7)}{\zeta(4)} \theta_{0}\right],
$$

where $E_{\mathrm{r}}=3 ! \zeta(4) \theta_{0}^{4}$ is the radiation energy. Equation (36) approximates the tail of the series with half of the $\theta_{0}^{2}$ term. Equations (32), (33) and (34) are compared with the numerical results. The energy rate change is calculated at early times since only then is the radiation field still Planckian.

In the first problem, a Planckian field at temperature $\theta_{0}=10^{-4}(51.1 \mathrm{eV})$ is upscattered by hotter electrons, initially at $\tau_{0}=10^{-2}(5.11 \mathrm{KeV})$. The specific heah, $c_{v}$, is chosen so that $\tau$ cools approximately one order of magnitude, $c_{v}=7.3617 \cdot 10^{-13}$. We compare $\tau, \theta, u_{\max }$, and the exchange rate in Table 4. Most results are accurate. The worst agreement is for the (initial) exchange rate. The numerical value is $5 \%$ high. 
In the downscattering problem, we initialize $\theta_{0}=10^{-2}(5.11 \mathrm{KeV}), \tau_{0}=10^{-4}(51.1 \mathrm{eV})$ and $c_{v}=6.4753 \cdot 10^{-3}$. For these values, $\tau$ increases nearly one order of magnitude. The comparisons appear in Table 5. Again, the accuracy is good, cf. the $\tau, \theta$ and $u_{\max }$ values. The only discrepancy is in the energy exchange rate. Both the analytic and numerical results are approximate. The series in Eq.(36) decays slowly. However, the numerical value is $42 \%$ low indicating that the scheme does not remove radiation energy fast enough.

\subsection{Bose-Einstein Equilibria}

In this section, stimulated scattering is included. The radiation field is initialized as in Eq.(28). The final distribution is given by Eq.(20). Photon number and total energy conservation give equations similar to Eqs.(30) and (31). However, the integrals of the final distribution are unwieldy. They are related to the $\Phi$ function, known only in terms of an infinite series ${ }^{130.325}$,

$$
\int_{0}^{\infty} d x x^{m} /\left(C e^{x}-1\right)=(m ! / C) \Phi(1 / C, m+1,1)=m ! \sum_{j=1}^{\infty} 1 /\left(C^{j} j^{m+1}\right) .
$$

Thus, the equations are

$$
\begin{gathered}
2 ! \zeta(3) \theta_{0}^{3}=(2 ! / C) \tau^{3} \Phi(1 / C, 3,1) \text { and } \\
c_{v} \tau_{0}+3 ! \zeta(4) \theta_{0}^{4}=c_{v} \tau+(3 ! / C) \tau^{4} \Phi(1 / C, 4,1) .
\end{gathered}
$$

The equations are made tractable by choosing initial parameters that lead to large values of $C$. In that case, $\Phi$ may be replaced by its leading term, 1. This leads back to Eqs.(30), (31), and (32) except $C$ is replaced by $1 / C$.

The energy exchange rate for the Fokker-Planck equation (first computed by Woodward ${ }^{14}$ ) is given in reference 9, Eq.(25),

$$
\frac{1}{4 E_{r}} \frac{d E_{r}}{d t}=\left(\tau-\theta_{0}\right)\left[1+\frac{5}{2} \tau-21 \frac{\zeta(5)}{\zeta(4)} \theta_{0}+\frac{15}{8} \tau^{2}-126 \frac{\zeta(5)}{\zeta(4)} \tau \theta_{0}+21^{2} \frac{\zeta(6)}{\zeta(4)} \theta_{0}^{2}+\cdots\right] .
$$

The expression is applicable only for a Planckian radiation field at temperature $\theta_{0}$. Hence, we compute the rate only at early times when $n \approx 1 /\left(e^{\gamma \theta_{0}}-1\right)$.

In the first problem, a Planckian field at temperature $\theta_{0}=10^{-6}(0.511 \mathrm{eV})$ is upscattered by hotter electrons, initially at $\tau_{0}=10^{-2}(5.11 \mathrm{KeV})$. The specific heat, $c_{v}$, is chosen so that $\tau$ cools approximately two orders of magnitude, $c_{v}=7.2871 \cdot 10^{-20}$. We compare $\tau, \theta, \mu_{\max }$, and the exchange rate as in the previous section. The comparisons, given in Table 6, are good; even the energy exchange rate compares well.

For the analogous downscattering problem (Bose Condensation), a Planckian field is surrounded by colder electrons $\left(\tau_{0}<\theta_{0}\right)$. This case, discussed by Youngs ${ }^{15}$ and reference 10 , leads to a dilemma. The inclusion of stimulated scattering implies that the equilibrium solution is given by Eq.(20), yet Eqs.(37) and (38) have no solution for $C \geq 1$ and $c_{\mathrm{v}}>0$. Physically, $n$, which at $t=0$ is given by Eq.(28), cannot retain the same number of photons, lose energy, and have a Bose-Einstein spectrum. The equilibrium is characterized by a Planckian at temperature $\tau<\theta_{0}$ and the extra photons form a $\delta$-function at $\gamma=0$. In a real medium, such photons would be absorbed. Nevertheless, this problem is interesting as it demands that the scheme remain stable.

For the analysis, we ignore number conservation and only use energy conservation,

$$
c_{v} \tau_{0}+3 ! \zeta(4) \theta_{0}^{4}=c_{v} \tau+3 ! \zeta(4) \tau^{4} \text {. }
$$

Given $c_{v}, \tau_{0}$ and $\theta_{0}$, we solve for $\tau$ using Newton's method. The numerical solution should at least find the Planckian distribution, Eq.(28), with $\theta_{0}=\tau$. The corresponding $u$ peaks at $\gamma_{\max }=2.8214 \ldots \tau$ with the peak value $\left(\gamma^{3} n_{p}\right)_{\max }=1.4214 \ldots \tau^{3}$.

Consider $\theta_{0}=10^{-2}(5.11 \mathrm{KeV}), \tau_{0}=10^{-4}(51.1 \mathrm{eV})$ and $c_{v}=6.4749 \cdot 10^{-5}$. These imply that $\tau$ should increase over one order of magnitude. The numerical solution undergoes a rapid spectral change. The energy density, $u$, separates into two peaks. The higher peak moves to the lowest frequencies modeling the previously mentioned $\delta$-function. The second peak moves to $\gamma_{P, \max }$ and represents the Planckian at $\theta=\tau$. The solution never comes to steady-state. As the run progresses, the magnitude of the first peak decreases; eventually it becomes comparable to the Planckian peak. If the run were to continue, the photons at $\gamma<1$ will be eliminated. This implies that the scheme does not conserve photons which contradicts the analysis of Sec.5. This 
number loss is significant; for the parameters chosen, the relative number lost is

$$
\left(N_{0}-N\right) / N_{0} \approx 1-2 ! \zeta(3) \tau^{3} / 2 ! \zeta(3) \theta_{0}^{3} \approx .999 \text {, }
$$

i.e. nearly all of the photons are lost. Despite this, the numerical solution retains the salient features of the expected equilibrium as we show in Table 7.

We believe that the loss of photons is due to finite precision of the computer. In this problem, the equilibrium solution consists of a superposition of a $\delta$-function and a Planckian ${ }^{15}$,

$$
n=\delta(\gamma)+\left(e^{\gamma \tau}-1\right)^{-1}
$$

where $\delta(\gamma)=0$ if $\gamma>\gamma_{1}$ for some $\gamma_{1} \ll 1$. The photons in $\delta(\gamma)$ have negligible energy, hence $\int \gamma^{3} n=3 ! \zeta(4) \tau^{4}$. However, since the number of photons is conserved, $N=\int \gamma^{2} n=\delta \gamma_{1}^{2} \Delta \gamma+2 ! \zeta(3) \tau^{3}=2 ! \zeta(3) \theta_{0}^{3}$. The initial parameters imply, $\tau^{3} \ll \theta_{0}^{3}$, hence most of the photons are in $\delta(\gamma)$. Approximating, $\Delta \gamma=\gamma_{1}$, it follows that $\delta$ is of order $\delta \approx N / \gamma_{1}^{3}$.

In the simulation there is a flow of photons to small $\gamma$. This results in a wide range in the magnitudes of the matrix coefficients in Eq.(24b). We can then approximate, $e_{j+1 / 2} \approx 1$ and $1-e_{j+1 / 2} \approx \Delta \gamma / \tau \approx \gamma / \tau$. Furthermore, $1+s n_{j}^{i} \approx n=N / \gamma_{j}^{3}$. Since $\alpha$ is of order 1 , we have

$$
b_{j}, a_{j} \rightarrow \Delta t \tau N,
$$

and for large $\Delta t$, the $\gamma_{j}^{2} \Delta_{j}$ term in the diagonal is swamped. For small $j$, Eqs.(24b) are no longer conservative and as the run progresses, there is a loss of photon number.

\section{Conclusion}

We have presented a collection of test problems and compared numerical results with analytic solutions. Such comparisons ascertain a method's credibility. After all, numerical solutions are only discrete approximations to analytic solutions of continuum equations. With the use of stable, positive methods, numerical results often look "good", but may be incorrect. An example is the FD energy transient in the flux-limited diffusion problem on the smooth mesh (Sec.3).

For the spatial diffusion schemes, Secs. 2 and 3 show that FE is more accurate than FD. For the strongly coupled problem of Sec.4, the diffusion operator is less important and the methods are of comparable accuracy.

Section 5 presented and evaluated a method to solve the Compton-Fokker-Planck equation. In nearly all of the problems, the method was very accurate. The worst performance was in the Bose-Condensation problem with the associated loss of photons. This is attributed to computer precision. In a realistic simulation, such a loss is unimportant as the lost photons have little energy and would be absorbed by the medium.

\section{References}

1. G. C. Pomraning, Radiation Hydrodynamics (Pergamon Press, Oxford, 1973).

2. Y. B. Zel'dovich and Y. P. Raizer, Physics of Shock Waves and High- Temperature Hydrodynamic Phenomena I (Academic Press, New York, 1966).

3. D. S. Kershaw, J. Comp. Phys. 39, 2, 375 (1981).

4. A. I. Shestakov, J. A. Harte, and D. S. Kershaw, J. Comp. Phys. 76, 2, 385 (1988).

5. A. I. Shestakov, J. Comp. Phys. 79, 1, 231 (1988).

6. C. D. Levermore and G. C. Pomraning, Astrophysical J. 248, 1, 321 (1981).

7. Y. B. Zel'dovich and Y. P. Raizer, Physics of Shock Waves and High- Temperature Hydrodynamic Phenomena II (Academic Press, New York, 1967), p. 664.

8. A. S. Kompaneets, Zh. Eksp. Teor. Fiz. $\underline{31}$ (U.S.S.R.), 876-885 (1956) and Soviet Physics Jetp 4 , 5, 730 (1957).

9. M. K. Prasad, A. I. Shestakov, D. S. Kershaw and G. B. Zimmerman, JQSRT 40, 1, 29 (1988) .

10. E. W. Larsen, C. D. Levermore, G. C. Pomraning, and J. G. Sanderson, J. Comp. Phys. $\underline{61}, 359$ (1985). 
11. M. Abramowitz and I. Stegun, Handbook of Mathematical Functions, Dover Publications, Inc., New York (1972).

12. A. I. Shestakov, D. S. Kershaw and M. K. Prasad, JQSRT 40 , 5, 577 (1988).

13. I. S. Gradshteyn and I. M. Ryzhik, Table of Integrals, Series, and Products, Corrected and Enlarged Edition, A. Jeffrey ed., Academic Press, Inc., Orlando (1980).

14. P. Woodward, Phys. Rev. D, 1, 10, 2731 (1970).

15. D. L. Youngs, Computation of the Time Variation of a Photon Distribution in a Compton Scattering Medium Report No. AWRE 44/92/28, Atomic Weapons Research Establishment, Berkshire, England (1984).

\section{Tables}

\begin{tabular}{|c|c|c|c|c|c|c|}
\hline mesh & scheme & $t$ & $u_{\max }$ & $u_{\min }$ & $f_{\max }$ & $f_{\min }$ \\
\hline $\mathrm{r}$ & $\mathrm{FD}$ & $2.663-3$ & $3.335-14$ & $3.438-14$ & $9.2-12$ & $3.5-12$ \\
\hline $\mathrm{s}$ & $\mathrm{FD}$ & $2.724-3$ & $1.08-13$ & $1.78-13$ & $2.66-12$ & $1.62-12$ \\
\hline $\mathrm{r}$ & $\mathrm{FE}$ & $2.695-3$ & $3.9297-14$ & $3.9242-14$ & $5.8888-12$ & $5.8806-12$ \\
\hline $\mathrm{s}$ & $\mathrm{FE}$ & $2.745-3$ & $3.3818-14$ & $3.3794-14$ & $5.0678-12$ & $5.0641-12$ \\
\hline
\end{tabular}

Table 1. Flux-limited diffusion problem. Range of energies $u$, and fluxes, $f \equiv-D \nabla u$, along $R_{1}$ at approx-imately $3 / 4$ of the light transit time. Notation: $r$ - random and $s$ - smooth meshes; $2.663-3$ is $2.663 \cdot 10^{-3}$.

\begin{tabular}{|c|c|c|c|c|c|c|c|}
\hline mesh & scheme & $u_{c}$ & $u_{\max }$ & $u_{\min }$ & $f_{c}$ & $f_{\max }$ & $f_{\min }$ \\
\hline $\mathrm{r}$ & $\mathrm{FD}$ & $6.9033-13$ & $6.6174-13$ & $6.6182-13$ & $1.0352-10$ & $0.7-10$ & $1.76-10$ \\
\hline $\mathrm{s}$ & $\mathrm{FD}$ & $n$ & $6.9009-13$ & $6.9007-13$ & $n$ & $1.083-10$ & $1.034-10$ \\
\hline $\mathrm{r}$ & $\mathrm{FE}$ & $6.89478-13$ & $6.89483-13$ & $6.89479-13$ & $1.03397-10$ & $1.03326-10$ & $1.03326-10$ \\
\hline $\mathrm{s}$ & $\mathrm{FE}$ & $n$ & $6.89523-13$ & $6.89521-13$ & $n$ & $1.03333-10$ & $1.03332-10$ \\
\hline
\end{tabular}

Table 2. Flux-limited diffusion problem. Comparison of analytic equilibrium energies and fluxes with numerical results along $R_{1}$. Same notation as in Table 1 .

\begin{tabular}{|c|c|c|c|c|c|c|c|}
\hline mesh & scheme & $t$ & $Z$ & $T$ & $t$ & $Z$ & $T_{e}$ \\
\hline r & FD & $6.9263+8$ & 1.078125 & $5.8703-3$ & $2.0126+9$ & 1.078125 & $9.8234-3$ \\
\hline S & FD & $6.8342+8$ & 1.078125 & $5.6346-3$ & $2.0035+9$ & 1.078125 & $9.8224-3$ \\
\hline r & FE & $6.8146+8$ & 1.093750 & $3.3530-3$ & $2.0015+9$ & 1.093750 & $9.6591-3$ \\
\hline S & FE & $6.9899+8$ & 1.093750 & $4.3962-3$ & $2.0100+9$ & 1.093750 & $9.6626-3$ \\
\hline
\end{tabular}

Table 3. Thermal wave problem. Exact solution of Eq.(15). Results of columns 3, 4, and 5 correspond to the curves of Fig.8. Results of columns 6, 7, and 8 correspond to the curves of Fig.9.

\begin{tabular}{|c|c|c|c|c|c|c|}
\hline result & $\tau$ & $C$ & $\theta$ & $\gamma_{W, \max }$ & $u_{\max }$ & $\left(d E_{r} / d t\right) / 4 E_{r}$ \\
\hline Analytic & $1.0079-3$ & $1.1741-3$ & $1.8291-4$ & $3.0237-3$ & $1.6160-12$ & $9.88-3$ \\
\hline Numerical & $1.0054-3$ & & $1.8292-4$ & $3.1622-3$ & $1.6089-12$ & $1.05-2$ \\
\hline
\end{tabular}

Table 4. Wien Upscattering problem. Initial values: $\theta_{0}=10^{-4}, \tau_{0}=10^{-2}, c_{\mathrm{v}}=7.3617 \cdot 10^{-13} . N_{\mathrm{v}}=50$, $\gamma_{1}=5.7736 \cdot 10^{-6}, \gamma_{j}^{2}=10^{-10}\left(10^{5}\right)^{(2 j-1)(50-1)}, j=2, \cdots, 50 . \gamma_{W, \max }=\gamma_{26}$. 


\begin{tabular}{|c|c|c|c|c|c|c|}
\hline result & $\tau$ & $C$ & $\theta$ & $\gamma_{W, \max }$ & $u_{\max }$ & $\left(d E_{r} / d t\right) / 4 E_{r}$ \\
\hline Analytic & $9.9235-4$ & $1.2301+3$ & $5.7618-3$ & $2.9771-3$ & $1.6159-6$ & $-7.7-3$ \\
\hline Numerical & $9.9213-4$ & & $5.7650-3$ & $3.1622-3$ & $1.6049-6$ & $-4.4-3$ \\
\hline
\end{tabular}

Table 5. Wien Downscattering problem. Initial values: $\theta_{0}=10^{-2}, \tau_{0}=10^{-4}, c_{v}=6.4753 \cdot 10^{-5}$. Same group structure as in Table 4.

\begin{tabular}{|c|c|c|c|c|c|c|}
\hline result & $\tau$ & $C$ & $\theta$ & $\gamma_{B_{\max }}$ & $u_{\max }$ & $\left(d E_{N} / d t\right) / 4 E_{r}$ \\
\hline Analytic & $1.0092-4$ & $8.5502+5$ & $3.2538-6$ & $3.0275-4$ & $1.6159-18$ & $1.0251-2$ \\
\hline Numerical & $1.0065-4$ & & $3.2540-6$ & $2.9673-4$ & $1.6140-18$ & $1.059-2$ \\
\hline
\end{tabular}

Table 6. Bose-Einstein Upscattering problem. Initial values: $\theta_{0}=10^{-6}, \tau_{0}=10^{-2}, c_{\mathrm{v}}=7.2871 \cdot 10^{-20}$. $N_{v}=70, \gamma_{1}=8.0333 \cdot 10^{-8}, \gamma_{j}^{2}=\left(1.3914 \cdot 10^{-7}\right)^{2}\left(7.1871 \cdot 10^{6}\right)^{(2 j-1 \gamma(70-1)}, j=2, \cdots, 70 . \gamma_{B, \max }=\gamma_{35}$.

\begin{tabular}{|c|c|c|c|c|}
\hline result & $\tau$ & $\gamma_{P, \max }$ & $u_{\max }$ & $\left(d E_{,} d d t\right) / 4 E_{r}$ \\
\hline Analytic & $1.1028-3$ & $3.1115-3$ & $1.9063-9$ & $-8.2309-3$ \\
\hline Numerical & $1.1028-3$ & $3.119-3$ & $1.705-9$ & $-4.9-3$ \\
\hline
\end{tabular}

Table 7. Bose Condensation problem. Initial values: $\theta_{0}=10^{-2}, \tau_{0}=10^{-4}, c_{\mathrm{v}}=6.4749 \cdot 10^{-5} . N_{\mathrm{v}}=70$, $\gamma_{1}=1.6290 \cdot 10^{-7}, \gamma_{j}^{2}=\left(2.8215 \cdot 10^{5}\right)^{2}\left(10^{6}\right)^{(2 j-1)(70-1)}, j=2, \cdots, 70 . \gamma_{P, \max }=\gamma_{48}$.

\section{Figure Captions}

1. "Random" computational mesh for linear diffusion test problem. Same mesh is used by FE scheme for flux limited problem; FD scheme compresses the ordinate to: $1 \leq R \leq 2$. In thermal wave problem, the domain: $0 \leq Z \leq 1,1 \leq R \leq 2$ is duplicated.

2. "Smooth" computational mesh for linear diffusion test problem. Same mesh is used by FE scheme for flux limited problem; FD scheme compresses the ordinate to: $1 \leq R \leq 2$. In thermal wave problem, the domain: $0 \leq Z \leq 1,1 \leq R \leq 2$ is duplicated.

3. Linear diffusion problem. Equilibrium energy per gram i.e. $u / 100$ vs. $Z$ along the outgoing boundary, $R=2$. Labels: 1 - FD scheme on random mesh, 2 - FD scheme on smooth mesh, 3 - FE scheme on random mesh, 4 - FD scheme on smooth mesh.

4. Linear diffusion problem. Equilibrium outgoing flux, $-D \nabla u$, vs. $Z$. Labels: 1 - FD scheme on random mesh, 2 - FD scheme on smooth mesh, 3-FE scheme on random mesh, 4 - FD scheme on smooth mesh.

5. Flux limited diffusion problem. FD contours of energy per gram i.e. $100 \cdot u$ at $t=(3 / 4) t_{c}$.

6. Flux limited diffusion problem. FE contours of energy per gram i.e. $100 \cdot u$ at $t=(3 / 4) t_{c}$.

7. Thermal Wave problem. FE nodal $T_{e}$ vs. $Z$ at $t=6.8146 \cdot 10^{8}$ for three different $K$ lines. Labels: $1: K=1$ or $R=2,2: K=16,3: K=33$ or $R=1$.

8. Thermal Wave problem. $T_{e}$ vs. $R$ at $Z=1.078125$ (FD) and at $Z=1.09375 F E$. Labels: 1 - FD random mesh, $t=6.9263 \cdot 10^{8} ; 2$ - FD smooth mesh, $t=6.8342 \cdot 10^{8} ; 3$ - FE random mesh, $t=6.8146 \cdot 10^{8} ; 4$ - FE smooth mesh, $t=6.9899 \cdot 10^{8}$.

9. Thermal Wave problem. $T$ e vs. $R$ at $Z=1.078125$ (FD) and at $Z=1.09375 F E$. Labels: 1 - FD random mesh, $t=2.0126 \cdot 10^{9} ; 2$ - FD smooth mesh, $t=2.0035 \cdot 10^{9} ; 3$ - FE random mesh, $t=2.0015 \cdot 10^{9} ; 4-\mathrm{FE}$ smooth mesh, $t=2.0190 \cdot 10^{9}$. 


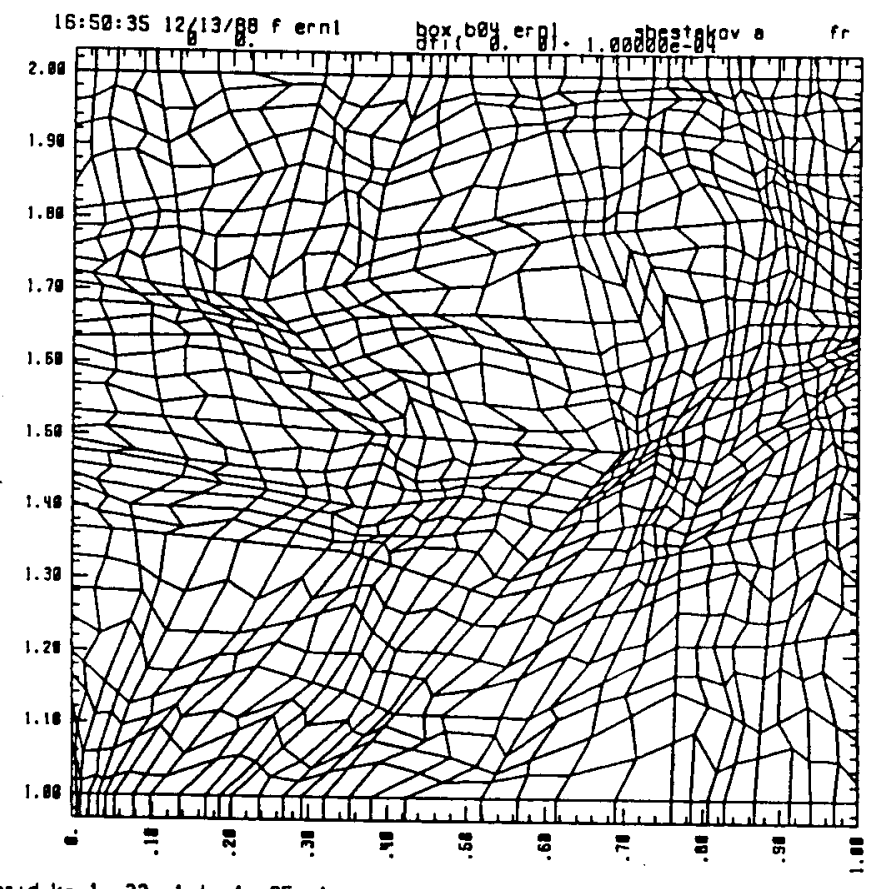

orid k= 1, 33. $11 \cdot 1,35,1$

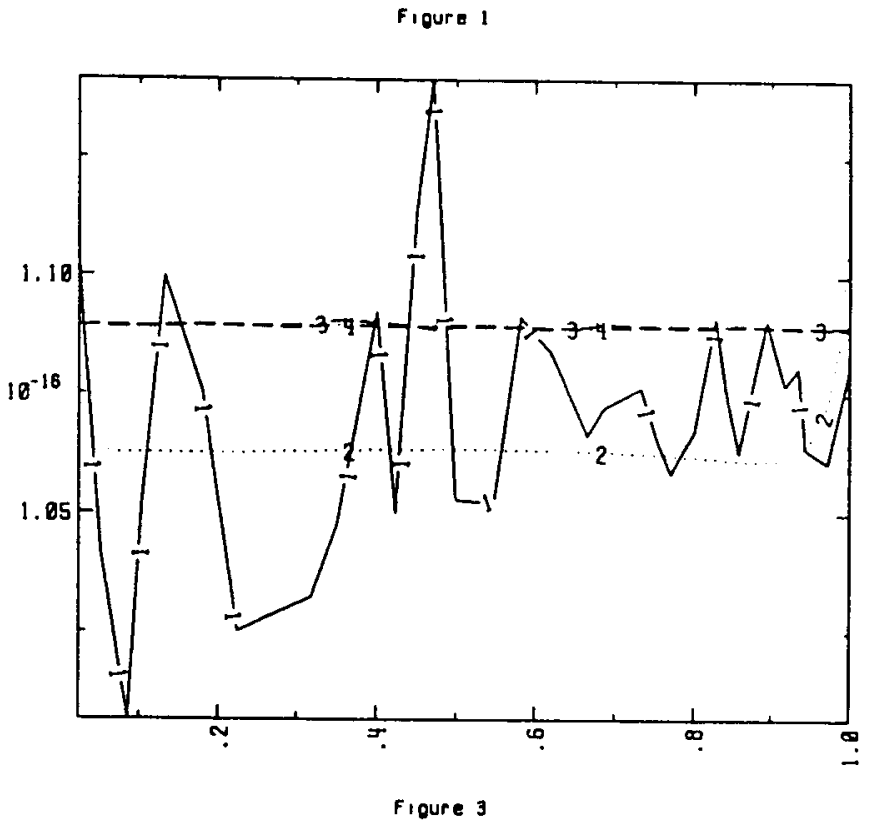

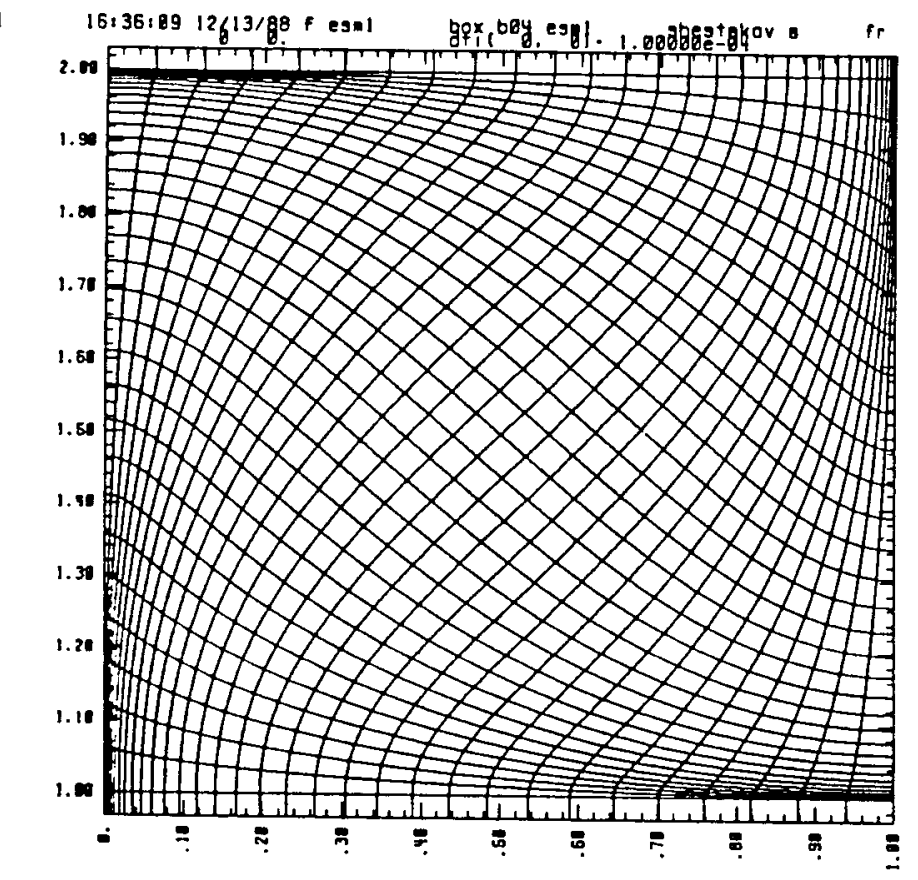

orid k=1.33, $11 \cdot 1,35,1$

Figure 2

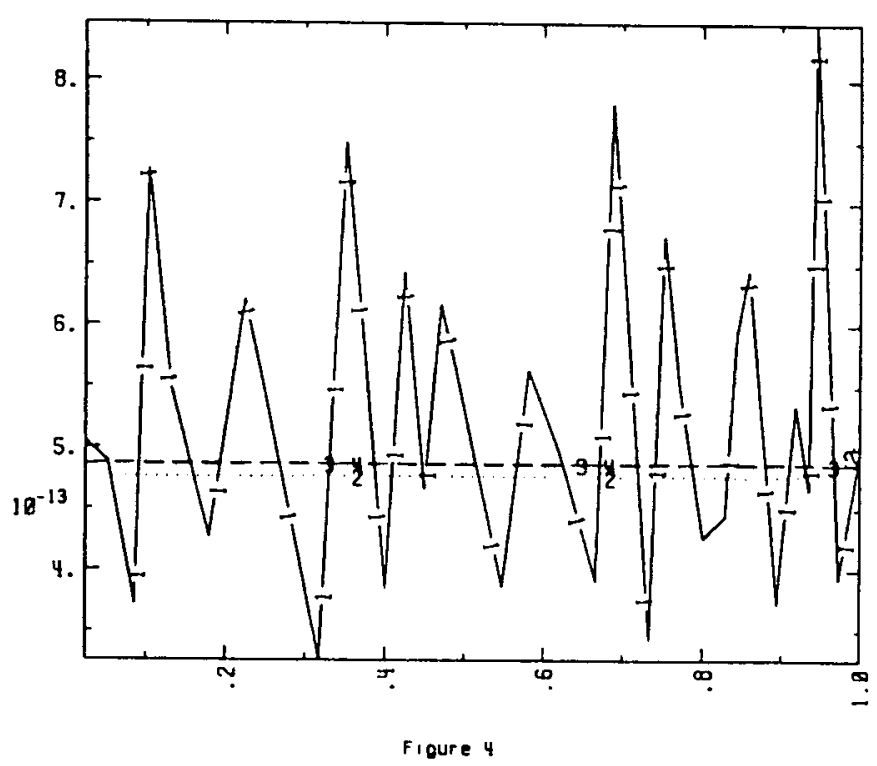




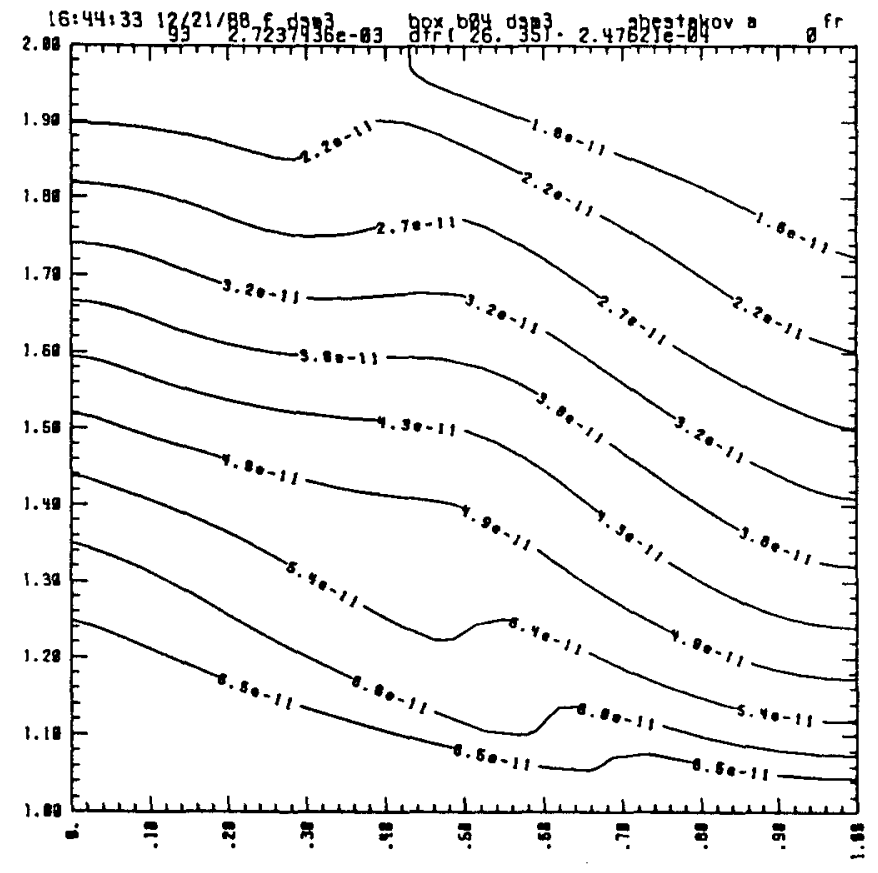

plotc er

Figure 5

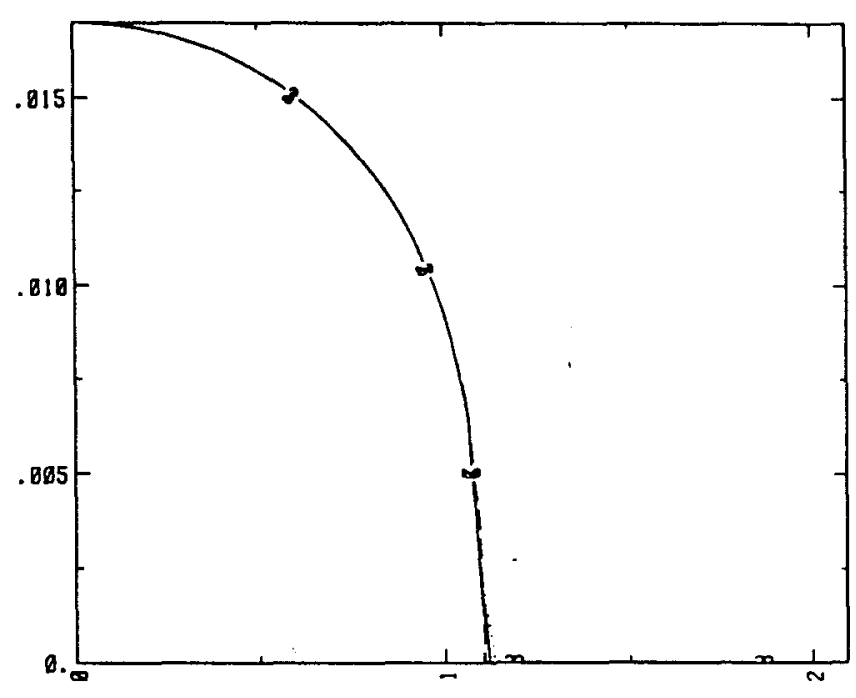

figure 7
16

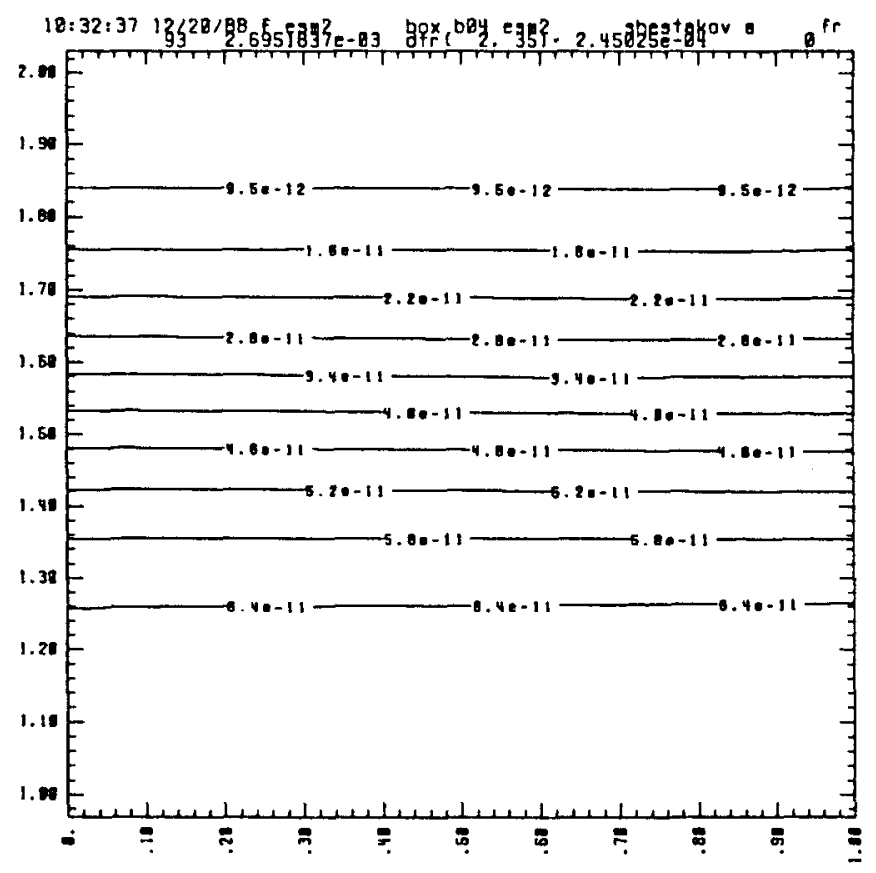

plotc er

Figure 6

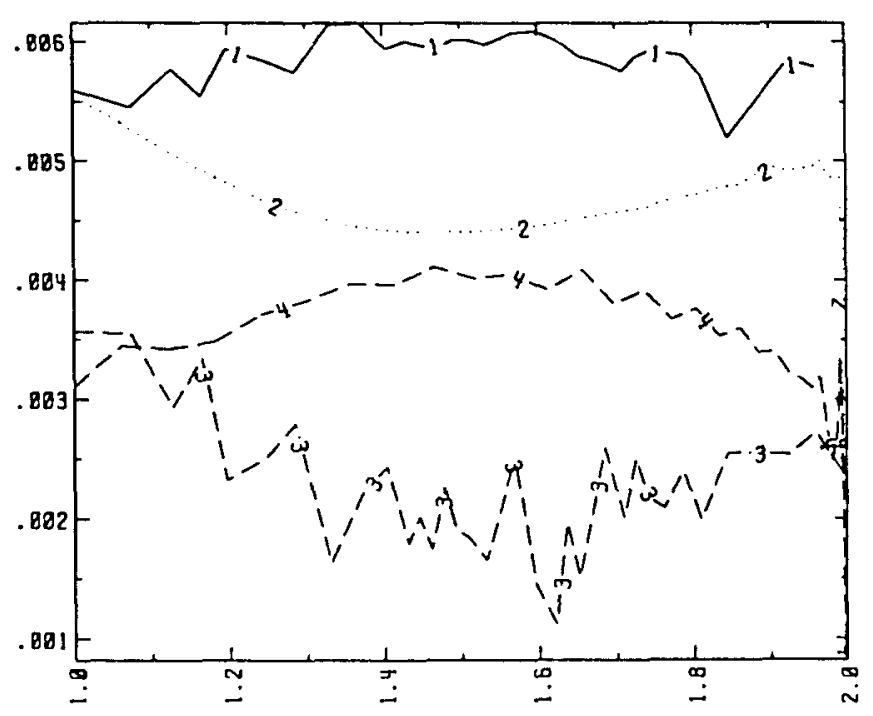

Figure 6

4

16

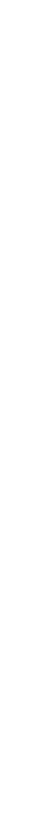




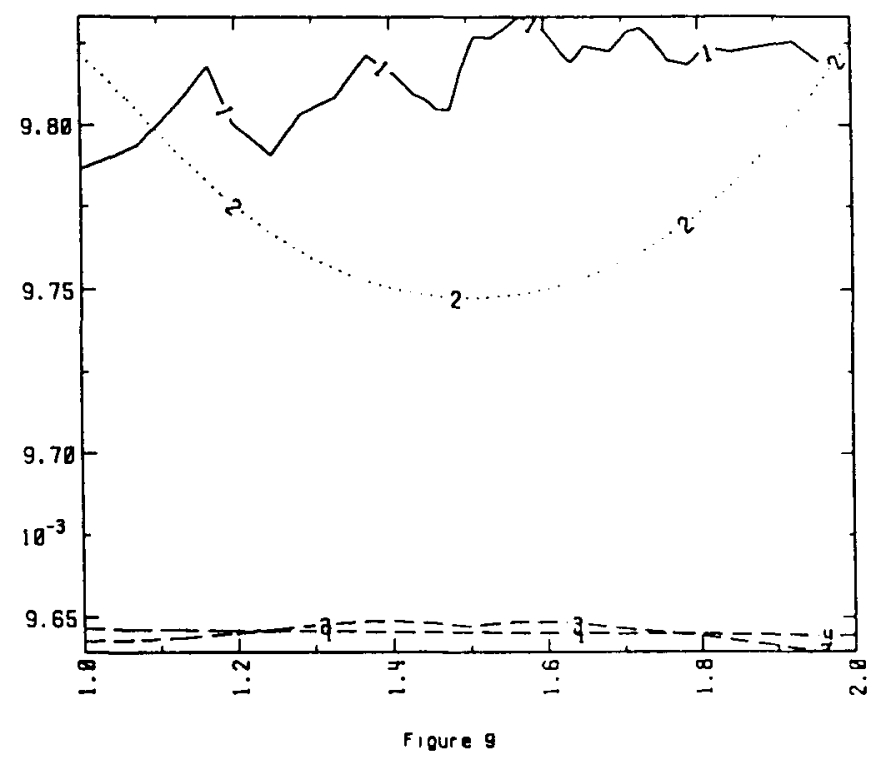




\section{Appendix A}

In the appendix we present the FORTRAN commands that generate a random mesh on the unit square, $0 \leq Z \leq 1,0 \leq R \leq 1$. The commands create a $\left(2^{\mathrm{nc}}+1\right) \times\left(2^{\mathrm{nc}}+1\right)$ mesh; other sizes are made by modifying the parameter "nc" on line 1. The parameter "a" in line 1 controls the "randomness"; $0 . \leq a \leq .5$ and $a=.5$ generates an orthogonal mesh. Lines $27,31,34,38,42$, and 43 have calls to a random number function "ranf," $0<\operatorname{ranf}<1$. Line 110 writes the mesh and the logical coordinates to unit 77 .

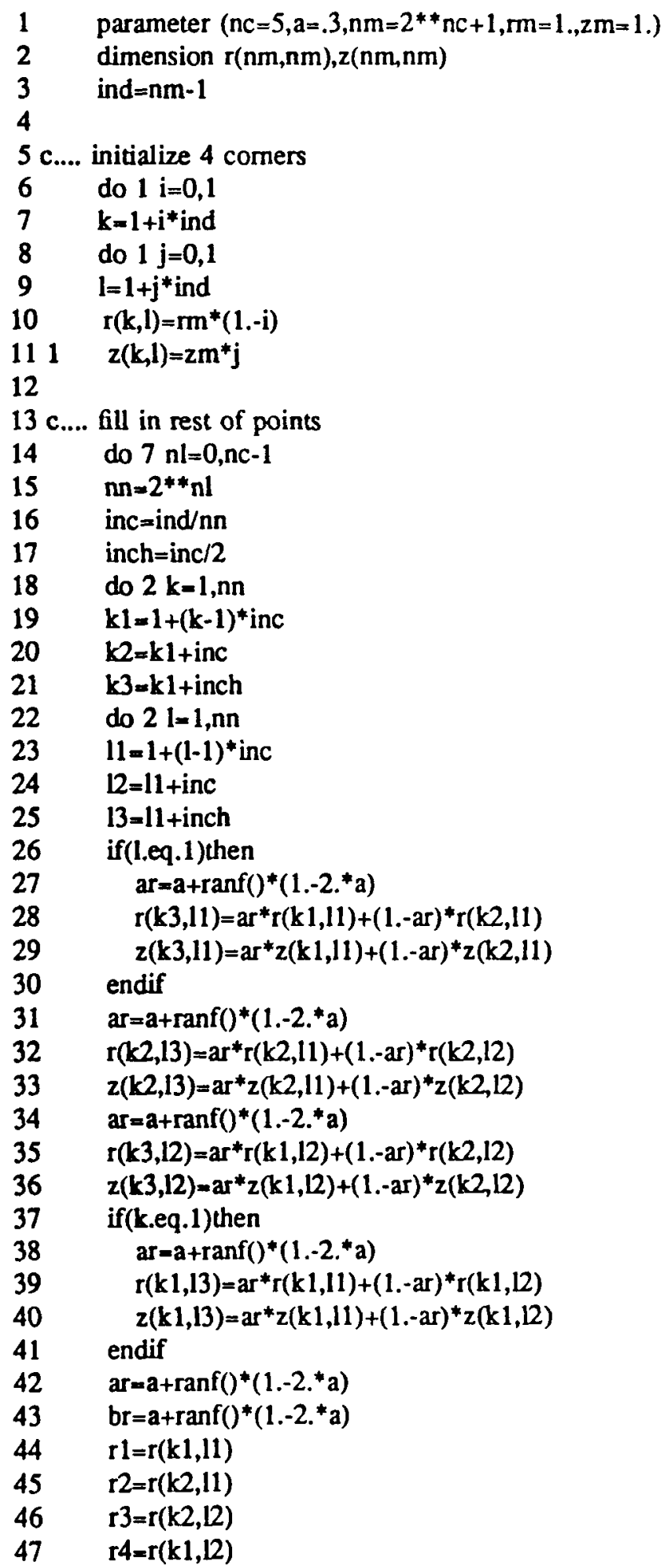




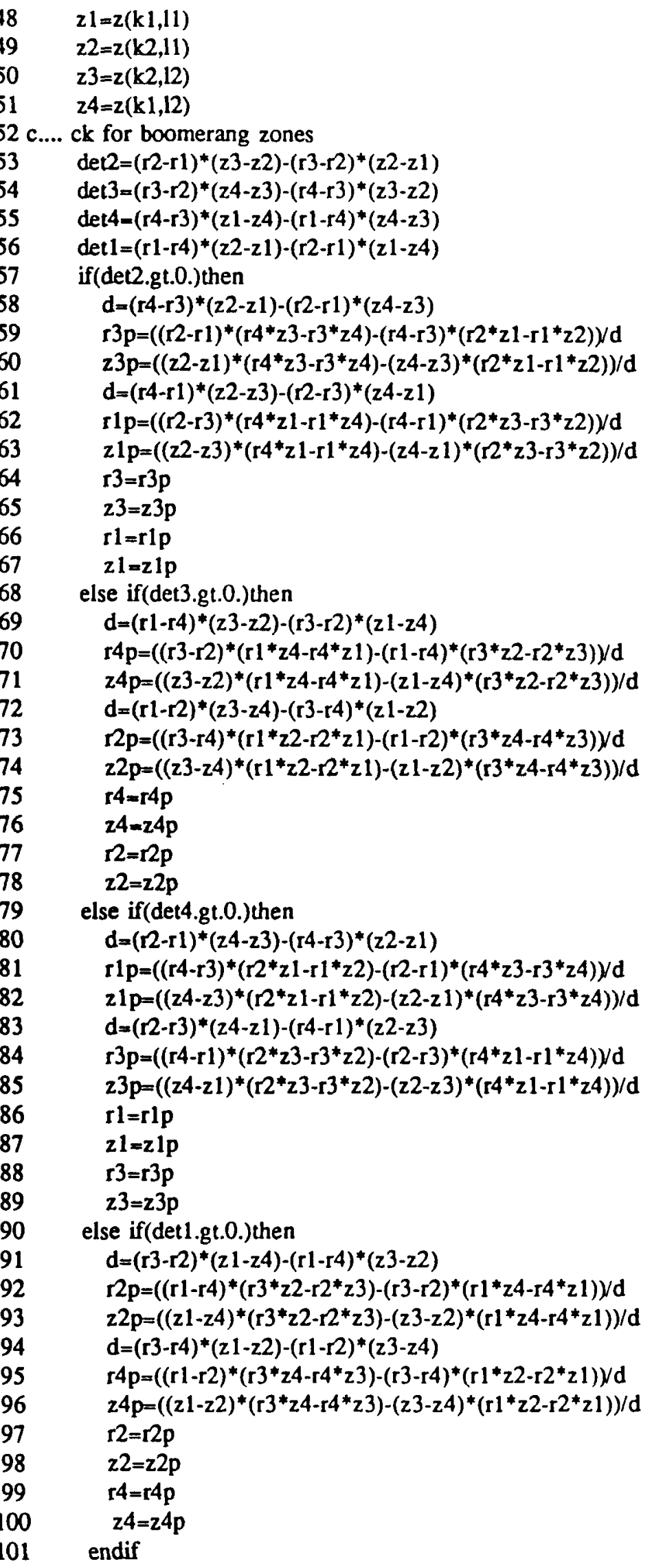


$102 \quad r(k 3,13)=a r^{*} b r^{*} r 1+a r^{*}(1 .-b r)^{*} r 4$

$1031+(1 .-a r) * b r * r 2+(1 .-a r)^{*}(1 .-b r)^{*} r 3$

$1042 \quad z(k 3,13)=a r^{*} b r^{*} z 1+a r^{*}(1 .-b r)^{*} z 4$

$1051+(1 .-a r) * b r * z 2+(1 .-a r) *(1 .-b r) * z 3$

106

107 c.... write $\mathrm{g}$ cards

108 do $3 \mathrm{k}=1, \mathrm{~nm}$

109 do $3 \mathrm{l}=1, \mathrm{~nm}$

1103 write $(77,101) \mathrm{k}, 1, \mathrm{k}, 1, \mathrm{r}(\mathrm{k}, 1), \mathrm{z}(\mathrm{k}, 1)$

111101 format("g ",4i3,2e24.13)

112

113 end 